\title{
Creating and Providing Predictions of Melanoma Outcome
}

\author{
Kenneth K. Tanabe, MD, Sebastian Jara, BSc, and James Michaelson, PhD \\ Massachusetts General Hospital and Harvard Medical School, Boston, MA
}

The development over the past few decades of large databases of cancer patients is providing physicians and their patients with the ability to make increasingly accurate and empirically based estimates of the chance of cancer survival. Two studies reported in this issue of the Annals of Surgical Oncology attest to the latest developments in the application of such a quantitative approach to the understanding of melanoma outcome.

The work described by Soong et al. is the latest in a 3decade long and heroic effort by this group to assemble large, multi-institutional, datasets of melanoma patients and then to make sense of these data. ${ }^{1}$ Their study in this issue of the Annals of Surgical Oncology describes the latest update in the Cox analysis of outcome for melanoma patients with localized disease and the development of a web-based calculator that makes these values available to physicians and patients. The predictive power of their model appears quite impressive and will be useful for guiding management recommendations as well as clinical trial design. Also, the ability to access such information over the Internet will be most welcome by practitioners. Of course, as Soong et al. would probably be the first to point out, this work represents the latest update in the everimproving art of such prediction. For example, the patient groups used in their model contain rather large bins into which they have sorted patients. One such example can be seen in their lumping together all patients with tumors of thicknesses 3.01-6.00 mm into a single group, which, by our own calculations, contains some patients with about a $25 \%$ chance of death and other patients with about a $45 \%$ chance of death even before other factors are taken into account. ${ }^{2}$ Similarly, lumping patients with $1.0-1.9 \mathrm{~mm}$ thickness melanomas in the same survival bucket seems

(C) Society of Surgical Oncology 2010

Published Online: 6 April 2010

K. K. Tanabe, MD

e-mail: ktanabe@partners.org counterintuitive in an era of massive datasets ever-growing in size, computerized analyses, and web-based interfaces. However, these quibbles are minor compared with their achievement of establishing and analyzing large datasets to allow for such predictions, and making them readily available over the Internet.

In this same issue of the Annals of Surgical Oncology, Bowles et al. provide information on the conditional survival for melanoma patients with node-positive disease. ${ }^{3}$ That is, they have calculated values for the likelihood of survival for those patients who have survived for some years after diagnosis. For most chronic illnesses, such as heart disease manifested as a myocardial infarction, once the acute period has passed, patients return to a low, but elevated likelihood of death to the same disease, whose probability increases gradually with time. ${ }^{4}$ Indeed, as we age, the instantaneous likelihood of death increases inexorably (and exponentially, as Gompertz showed more than a century ago), a doleful fact that demographers call, in operatic tone, "the force of mortality." Cancer differs from this more general case because while the risk of death usually rises in the few years after diagnosis, that risk of death will eventually begin to decline, and ultimately reach very low levels, such that patients may functionally be considered "cured." Bowles et al. demonstrated this in their analysis of conditional survival for node positive melanoma patients. They found for those patients who have survived disease free for 5 years, that disease-specific and disease-free survival rates for the next 5 years increase noticeably. This is welcome news, which physicians can provide to patients who have lasted through the highestrisk period in the first few years after diagnosis. This concept is also somewhat intuitive, as oncologists will always spot the negative slope of a survival cure-as well as the point at which the slope begins to flatten out.

Our group has also been at work on the development a mathematical framework for comprehending cancer outcome, together with the assembly of very large databases 
on cancer patients, and the creation of web-based calculators for predicting outcome for colon cancer, renal cell carcinoma, breast carcinoma, and melanoma (http://www. CancerMath.net). ${ }^{5,6}$ Our analysis, outlined in a technical report available at the CancerMath.net website, indicates that the outcome predictions provided by both our melanoma calculator, and that of Soong et al., accurately predict the chance of survival, as tested against the Surveillance, Epidemiology, and End Results (SEER) melanoma national dataset provided by the National Cancer Institute. ${ }^{7}$ However, as noted previously, the calculator developed by Soong et al. sorts patients into rather large bins, which contain mixes of patients with widely disparate risks of death. The CancerMath.net website also provides conditional survival calculators, including one for melanoma.

Clearly, the work exemplified in the reports by Bowles et al. and Soong et al. represent an ongoing effort to develop more accurate mathematical models, and progressively more useful web-based tools, for predicting cancer outcome. Now that the science of prediction has arrived for melanoma, attention will next need to turn to finding ways to provide this information in the most useful manner possible. The form in which such survival information is presented to physicians and their patients, that is, the way in which such predictions are "framed" can have a dramatic impact on how such information is used. In the classic 1982 study by McNeil et al., experienced physicians who were told that their patients can expect a $10 \%$ perioperative mortality rate arrived at very different treatment plans than equally experienced physicians who were told that their patients would experience a $90 \%$ perioperative survival rate. ${ }^{8}$ Attention will also need to be applied to how such information is presented to patients in a way that is most helpful to them. However, this is a most welcome problem, made possible by the capacity to provide such predictions accurately, as demonstrated by the work of Bowles et al. and Soong et al. ${ }^{1,3}$

\section{REFERENCES}

1. Soong S-J, Ding S, Coit D, Balch CM, Gershenwald JE, Thompson JF, et al. Predicting survival outcome of localized melanoma: An electronic prediction tool based on the AJCC melanoma database. Ann Surg Oncol. 2010. doi:10.1245/s10434-010-1050-z.

2. Michaelson JS, Chen LL, Silverstein M, Mihm MV, Jr., Sober AJ, Tanabe KK, et al. How cancer at the primary site and in the nodes contributes to the risk of cancer death. Cancer. 2009;115:5095107.

3. Bowles TL, Xing Y, Hu C-Y, Mungovan KS, Askew RL, Chang GJ, et al. Conditional survival estimates improve over 5 years for melanoma survivors with node-positive disease. Ann Surg Oncol. 2010. doi:10.1245/s10434-010-1051-y.

4. Gulliksson M, Wedel H, Köster M, Svärdsudd K. Hazard function and secular trends in the risk of recurrent acute myocardial infarction; 30 years of follow-up of more than 775000 incidents circulation. Circ Cardiovasc Qual Outcomes. 2009;2:178-85.

5. Michaelson JS,Chen LL, Silverstein M, Cheongsiatmoy JA, Mihm MV, Jr., Sober AJ, et al. Why cancer at the primary site and in the nodes contributes to the risk of cancer death. Cancer. 2009;115: 5084-94.

6. Chen LL, Nolan M, Silverstein M, Mihm MV, Jr., Sober AJ, Tanabe KK, et al The impact of primary tumor size, nodal status, and other prognostic factors on the risk of cancer death. Cancer. 2009;115:5071-83.

7. Jean RA, Chen LL, Jara SM, Bush DM, Tanabe KK, Sober A, et al. Technical Report No. 11, Comparative effectiveness calculators for predicting melanoma death, Laboratory for Quantitative Medicine, August 19, 2009. Available at http://www. lifemath.net/cancer/about/techreports/index.php.

8. McNeil, BJ, Pauker, SG, Sox, HC, Tversky, A, On the elicitation of preferences for alternative therapies. $N$ Engl J Med. 1982;306: 1259-62. 